\title{
Síndrome de Korsakoff: Determinación de patrones asociados al consumo del alcohol en estudiantes de nivel superior de la UAEH-ESH
}

\author{
Korsakoff syndrome: determination of patterns associated with the consumption of alcohol in \\ higher level students of the UAEH-ESH
}
Zaida Burgos-Ostoa a , Juan Hernández-Medina ${ }^{a}$, Eidy L. Hernández-Hernández, Ángeles Cruz- Lara $^{a}$, Oscar Popoca-Reyes ${ }^{a}$, Marco Antonio Hernández-Bedolla ${ }^{b}$

\begin{abstract}
:
Korsakoff syndrome is a disease caused by alcoholism and the most frequent cause of amnesia. Memory and learning are especially affected. The alterations manifest as anterograde amnesia (create new memories). There may also be retrograde amnesia (difficulty accessing memories of the past). Globally and mainly in the Huasteca region, alcohol consumption is a Public Health problem. A population sensitive to this phenomenon are teenagers or young adults. Due to the high consumption of intoxicating beverages in Huejutla de Reyes Hidalgo city, this research will be carried out to identify patterns associated with alcohol consumption and detect possible consequences in memory and learning in higher-level students of the Autonomous University of Hidalgo State - Huejutla High School.
\end{abstract}

Keywords:

Keywords: Alcoholism, Amnesia, Memory, Learning

\section{Resumen:}

El Síndrome de Korsakoff es una enfermedad ocasionada por el alcoholismo y causa más frecuentes de amnesia. Se ven afectados especialmente la memoria y el aprendizaje. Las alteraciones se manifiestan como una amnesia anterógrada (crear recuerdos nuevos). También puede llegar a presentarse amnesia retrógrada (dificultad de acceso a recuerdos del pasado). A nivel mundial y principalmente en la región de la Huasteca el consumo de alcohol es un problema de Salud Pública. Una población sensible a este fenómeno son los adolescentes o adultos jóvenes. Debido al alto consumo de bebidas embriagantes en el municipio de Huejutla de Reyes Hidalgo se realizará esta investigación para identificar patrones asociados al consumo de alcohol y detectar las posibles consecuencias en la memoria y el aprendizaje en estudiantes de nivel superior de la Universidad Autónoma del Estado de Hidalgo Escuela Superior Huejutla.

Palabras Clave:

Alcoholismo, amnesia, memoria, aprendizaje 


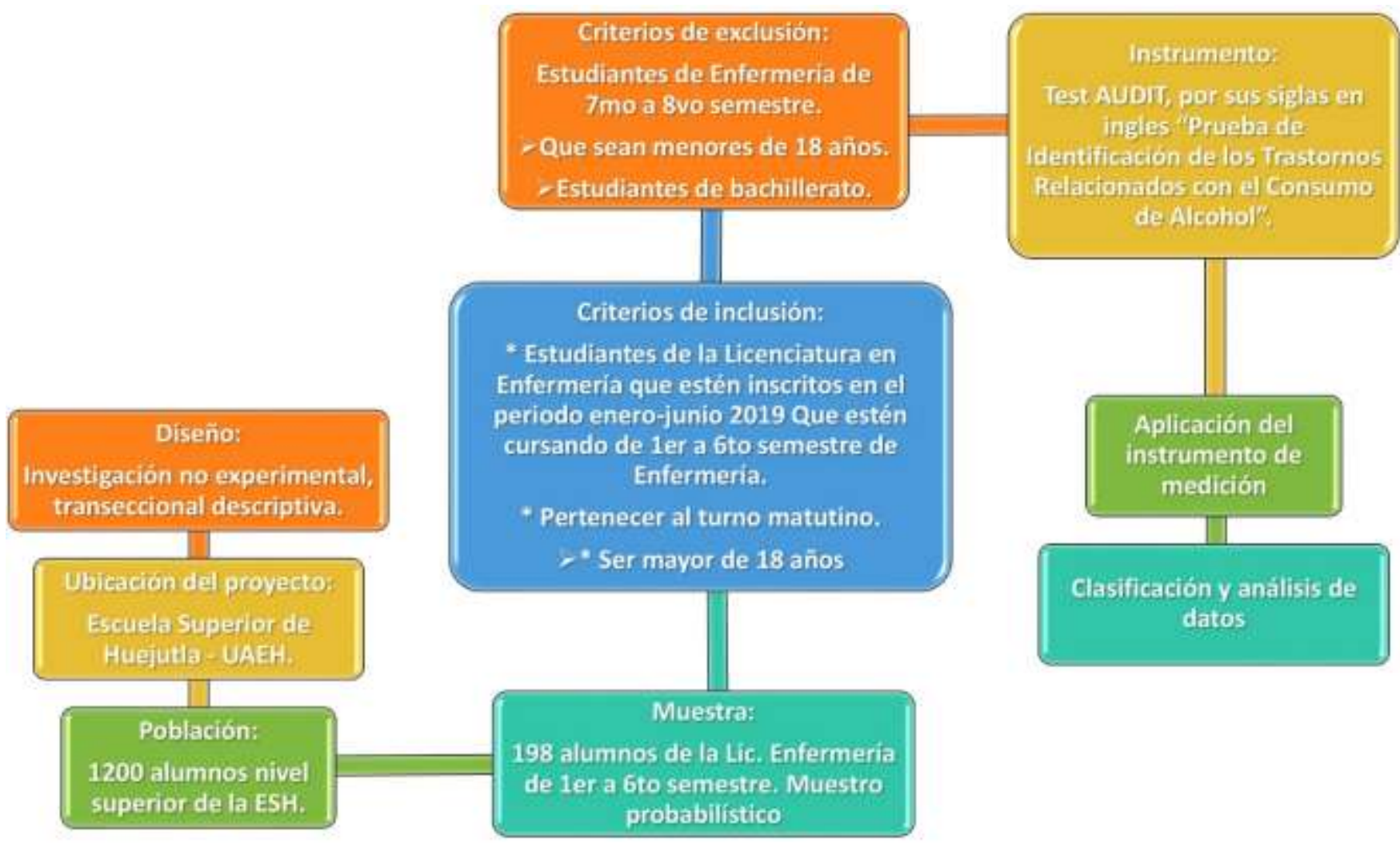

La región de la Huasteca es una zona donde el consumo de alcohol es un problema grave de salud pública, observándose que la comunidad estudiantil es la principal población vulnerable a esta complicación; debido a que es muy fácil encontrar algún motivo para poder consumir bebidas alcohólicas, además de su fácil adquisición. De tal manera, nos interesa determinar patrones asociados al consumo de alcohol en estudiantes del nivel superior de la Escuela Superior de Huejutla, teniendo como primer indicio una prueba piloto en estudiantes de la Licenciatura de Enfermería que cursan en el periodo enero-junio 2019. La finalidad de este estudio es determinar mediante la evaluación de los patrones asociados al consumo de alcohol que consecuencias pueden tener en su rendimiento académico la comunidad estudiantil de esta casa de estudios.

\section{REFERENCIAS}

(1) Karam, T.M., Echevarría Zuno, D., Sandoval Castellanos, D. J., González Izquierdo, D., Aguilar Sánchez, D., \& Morales Rojas, D. (2011). Diagnóstico y Tratamiento de la Encefalopatía de Wernicke o síndrome de Korsakoff. (D. d. Clínica, Ed.) Recuperado el 3 de septiembre de 2018, de http://www.imss.gob.mx/profesionales/guiasclinicas/gpc.htm.

(2) Hernández Armas, D. M., Sánchez Sigler, M. R., Ríos Rodríguez, M., \& Baigorri Solange, J. (2010). Alcoholismo y adolescencia. policlínico universitario "Luis li tregent", 1004-105.

(3) M. Womble, D. (2013). Introducción a la enfermería en la salud mental. Barcelona, España: Wolters Kluwer/ Lippincott Williams \& Wilkins.

(4) F. Babor, T., Higgins-Biddle, J., Saunders, J., \& Monteiro, M. (2001). AUDIT Cuestionario de Identificación de los Trastornos debidos al Consumo de Alcohol. Obtenido de AUDIT Cuestionario de Identificación de los Trastornos debidos al Consumo de Alcohol. 